CLAWAR 2018: 21st International Conference on Climbing and Walking Robots and the Support Technologies for Mobile Machines, Panama City, Panama, 10-12 September 2018

\title{
DEVELOP A SENSOR BASED ROBOT FOR RAILWAY INSPECTION
}

\author{
Nathan Western; Shyamal Mondal \\ Department of Engineering, London South Bank, 103 Borough Road \\ London, SE1 OAA, England
}

\begin{abstract}
This project produced a sensor based robot capable of testing a section of railway track, using both magnetic flux and visual inspection. Surface quality inspection is performed by a probe consisting of a number of Hall effect sensors. Visual inspection is performed by a camera connected to the robot. A replica rail section was also created for the purposes of calibrating and demonstrating the robot. The hardware and software developed successfully collected video data and magnetic flux readings. The output of the hall effect sensor array was processed in MATLAB producing a heat-map. The shape and depth of artificially induced defects were successfully displayed.
\end{abstract}

\section{Introduction}

In this project a robot was created to provide non-destructive testing (NDT) for rail. It aimed to provide a service comparable to a fully staffed test. It is hoped this will be a step towards more regular testing by being convenient and cheap to use, resulting in a safer railway service. A variety of NDT methods were considered and evaluated, and two were selected and incorporated into a functional testing robot that can detect common flaws in rail track.

Rail testing and maintenance is essential to keeping Britain's extensive network of train track operational. 1.7 billion journeys were made in 2016 accounting for $8 \%$ of all passenger travel and over $10 \%$ of all freight moved [1]. Maintenance costs Network Rail $£ 1.3$ billion a year according to latest available figures from 2016-2017 [2]. It is still primarily carried out by technicians and engineers on site. Not only is this costly, it also limits the time at which maintenance can be performed to ensure the safe working conditions of the engineers. This creates problematic scheduling conflicts.

Considering this, the project aimed to determine whether automation could reduce the costs of routine rail inspection or increase the effectiveness of NDT for rail. This should be achieved using a mobile testing platform capable of utilising multiple testing methods. 


\section{Rail Inspection Robot Design}

The platform was designed to enable movement across the rail and contact between the rail and the sensor array. A single rail crawler design was selected.

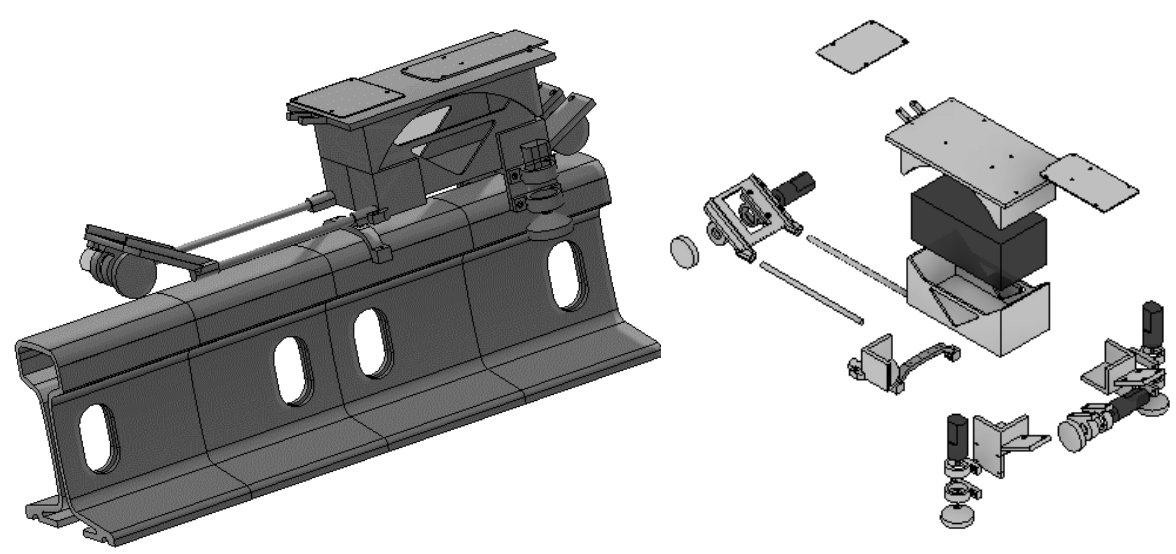

Figure 1a - Assembly of Robot and Track

Figure 1b - Exploded View of Robot

This used wheels on the top and sides of the rail to travel across one rail and perform an inspection. This design supported visual and magnetic flux inspection with the optimal cost, time and materials usage.

The robot was initially designed to move at a speed of $0.1 \mathrm{~m} / \mathrm{s}$ to ensure the sensors could take accurate readings. It would be hoped that the speed could be increased in future developments, but at this feasibility stage it was more important to achieve accuracy to prove the concept.

The sensor array consisted of seven Hall effect sensors. The Arduino Mega board powered the sensors and collected the output signal. This was sent via serial out to the Raspberry Pi for storage. Neodymium magnets were used to magnetise the rail, and the sensors then measured any change in flux caused by defects. The sensors were positioned an equal distance from each other and the magnets.

The robot comprises of two sub-assemblies. The lower part of the chassis holds the motors, battery and sensor array. The upper chassis, which sits on top of the lower, contains the microprocessors, power supply for the boards and the 
camera. The chassis was printed in PLA plastic using Fused Filament Fabrication techniques.
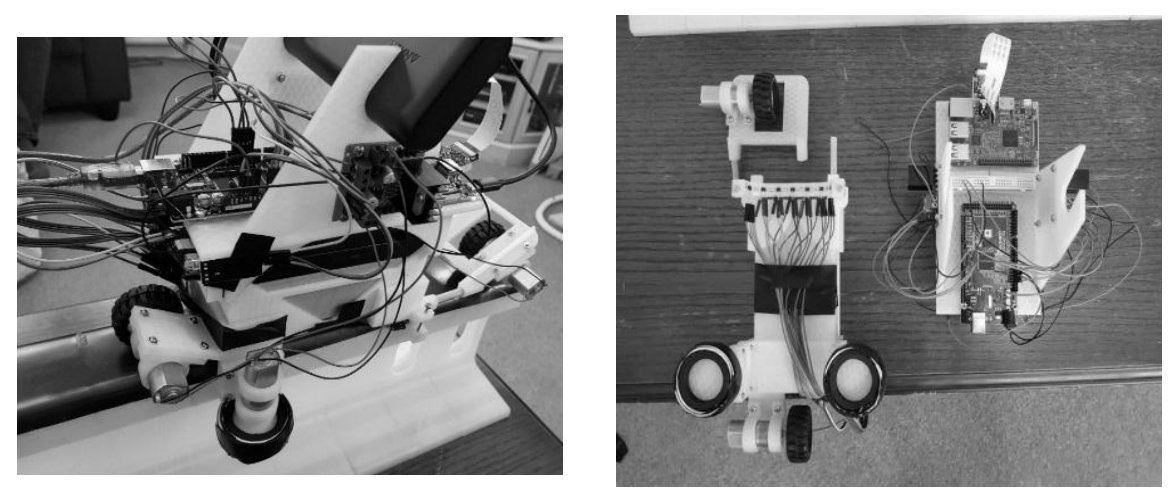

Figure $2 \mathrm{a}-\mathrm{A}$ robot carried out inspection on the rail track

Figure $2 b$ - Disassembled Robot

\section{Robot Controller Software Design}

The Arduino takes readings from the sensor array at an interval of $1 \mathrm{~ms}$. This data is then sent via Serial.print to the Raspberry Pi for logging as a .csv file. The Arduino was also responsible for managing the movement of the robot. An infrared sensor detects the presence of obstacles in the path of the robot and sends a signal to the motors, only if the path is clear.

The Raspberry Pi was instructed to collect video and serial data using the command line over SSH. The video was collected using the raspivid command. The serial data was collected using the grabserial library.

\section{Data Processing Method}

The Hall effect sensor data was analysed using MATLAB. A program was produced to calculate the average gauss reading for each sensor, and then construct a matrix of the difference between the average values and the results. A contour plot was chosen to represent this data because it displays the change in Gauss readings visually. Higher difference in Gauss values was shown as a darker colour, indicating a deeper defect. 

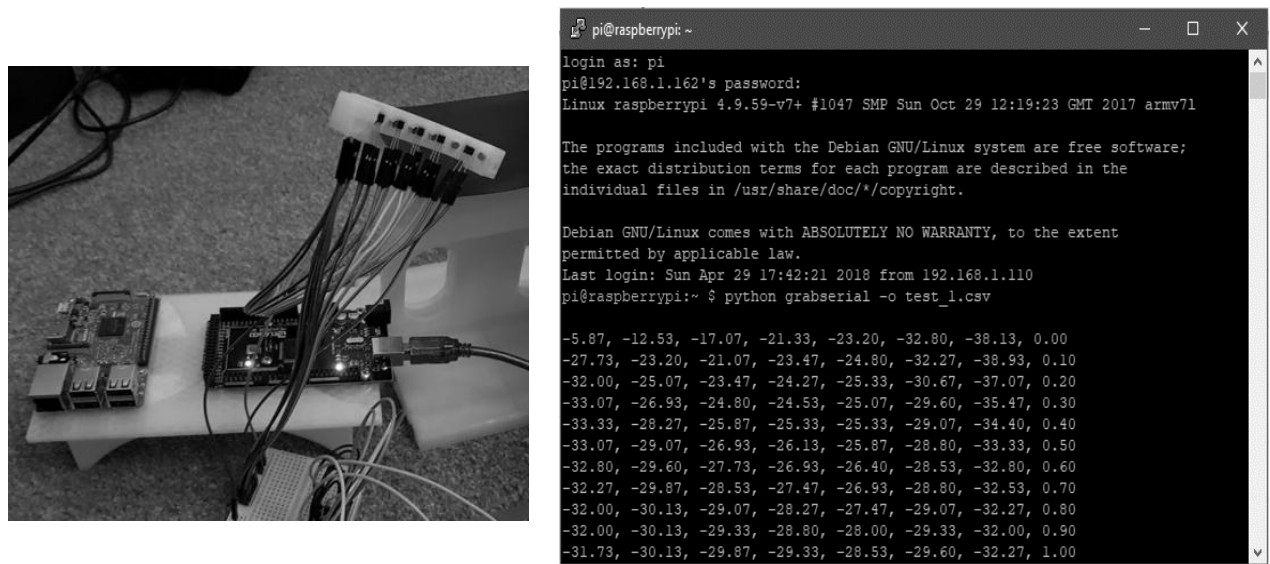

Figure 3 - Using the Grabserial Command to Collect Data from the Arduino

\section{Results and Discussion}

The robot collected data from the Hall effect sensors and camera as it travelled the length of the rail section. The rail contained 5 defects of various size and depth. These represented common rail faults; severe detail fracture (at $590 \mathrm{~mm})$, spalling as a result of rolling contact fatigue $(515 \mathrm{~mm})$, two small head checking defects $(380 \mathrm{~mm}$ and $435 \mathrm{~mm})$, and finally a gauge corner crack with divergence $(212 \mathrm{~mm})$. Multiple tests were conducted to determine the ideal distance between the Hall effect sensor array and the track.

\subsection{Test $1-0.5 \mathrm{~mm}$ Sensor Height from Rail Surface}

The initial test results are shown in Figure 4 after being processed by the MATLAB program. The readings were taken with the sensor a distance of approximately $0.5 \mathrm{~mm}$ from the rail surface. Although there is some interference caused by the uneven surface all the defects are shown. Especially clear is the spalling type defect visible at reading 60 . The sensor array was raised to a height of $1.5 \mathrm{~mm}$ for the next test to try to remove some of the interference. 

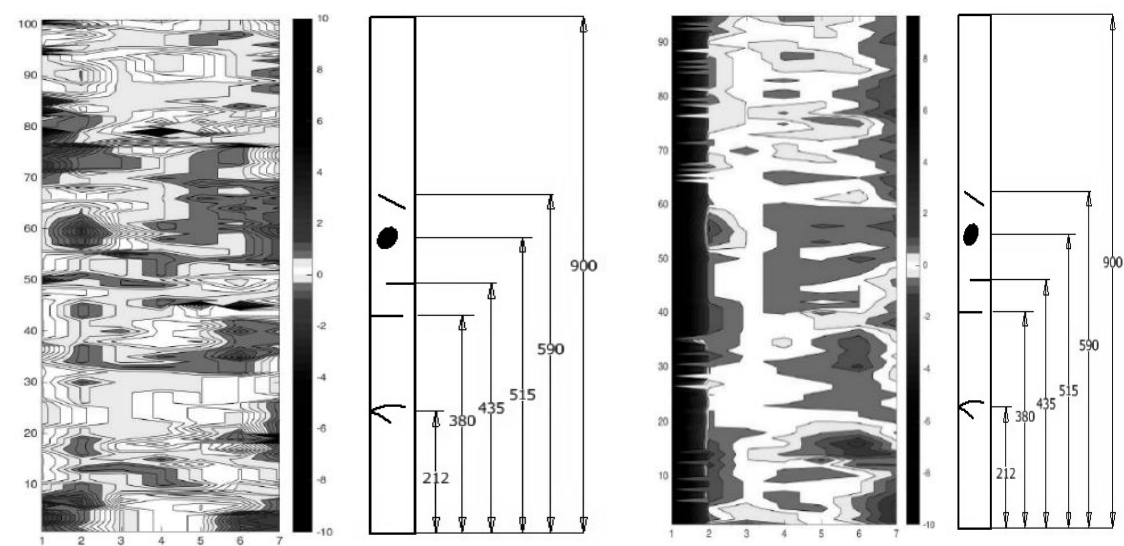

Figure 4 - Test 1 Results for 0.5mm Sensor Height Figure 5 - Test 2 Results for 1.5mm Sensor Height

\subsection{Test 2 - 1.5mm Sensor Height from Rail Surface}

During test 2 the sensor array was raised too far and did not make proper contact with the rail. This lead to the results being harder to interpret. The robot also slipped towards the end of the test, causing the jagged pattern visible from reading 70 onwards in Figure 5. The sensor was lowered to $1 \mathrm{~mm}$ for the next test.

\subsection{Test 3 - 0.7mm Sensor Height from Rail Surface}

Test 3 produced a clearer image of the test rail than test 2, as shown in Figure 6 . The divergent crack at $212 \mathrm{~mm}$ is more clearly shown from readings 5-15. The deepest crack at $590 \mathrm{~mm}$ is now very clear between readings $60-70$. This did cause some distortion in the area by readings 50-60, making the spalling defect harder to see. The sensor was raised just $0.2 \mathrm{~mm}$ for test 4 to compensate for this. 

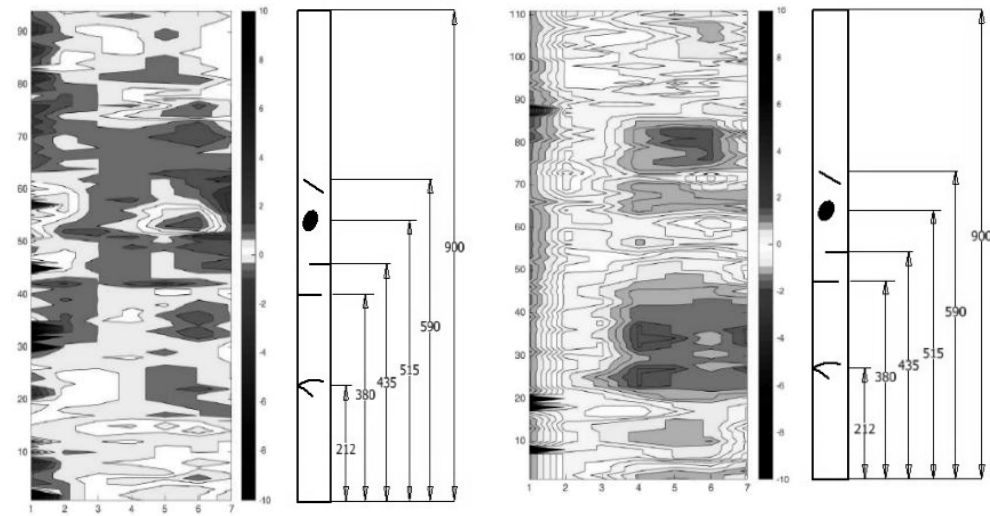

Figure 6 - Test 3 Results for $0.7 \mathrm{~mm}$ Sensor Height Figure 7 - Test 4 Results for 0.9mm Sensor Height

\subsection{Test 4 - 0.9mm Sensor Height from Rail Surface}

Figure 7 shows the results taken during test 4 . The dark orange shape at readings $10-15$ show a change in Gauss of 1.75 . This was caused by the gauge corner crack at $212 \mathrm{~mm}$, and the shape of the defect is represented. The two cracks at $380 \mathrm{~mm}$ and $435 \mathrm{~mm}$ are clearly shown at reading 25 and 35, with an indication of the shape and depth of the cracks. The spalling defect is less clear, but still visible in the readings at position $65-70$. Finally, the deep crack at $590 \mathrm{~mm}$ is shown at reading 80 , with indication of depth.

\subsection{Video Inspection Data}

Visual data was also collected by the robot using the Raspberry Pi camera. Stills from the video are shown in figures $8 \& 9$ below. This is the ferromagnetic top surface of the PLA replica rail section. When a defect is detected by the sensor array the video can be examined to further determine the nature of the faults. This also reduces erroneous readings, for example the sensor array travelling over organic debris such as leaves. 

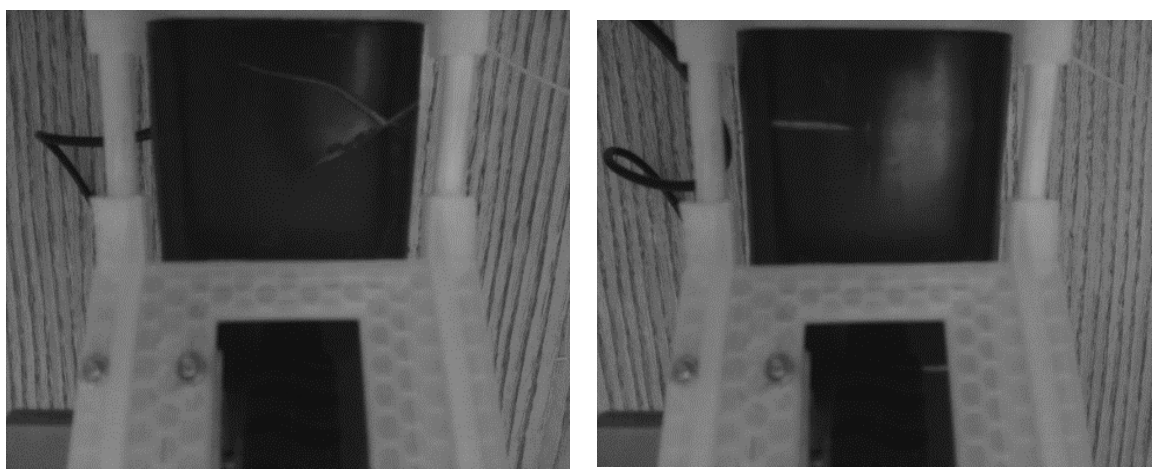

Figures 8 \& 9 - Images of Cracks in the Gauge and Head Region

\section{Conclusions and Recommendations for Future Work}

\subsection{Recommendations for future work}

The slow speed of the robot was identified as a weakness during the project. If the speed could not be increased this could be compensated for by scheduling tests for small parts of the rail at one time. A study of railway maintenance [3] stated that a busy European railway might have inspection intervals of 4 months. Assuming testing being conducted solely during the working week, one robot could be scheduled to test each $115.2 \mathrm{~km}$ of rail in a 4-month period.

The distance the sensor is operating from the rail has a large impact on the results. The design would benefit from the addition of a thumb screw system to make sensor height adjustments easier. The addition of small wheels or rollers to the bottom of the sensor would also increase sensor stability.

\subsection{Conclusions}

A robot capable of traversing railway track was developed, using a sensor array to conduct non-destructive testing of the rail. Additionally, the robot uses visual inspection to conduct a test of a section of track. This ensures a greater degree of accuracy and more conclusions about any defects can be drawn. The digital data was suitably interpreted, producing an image of the rail condition. Achieving this level of inspection using an automated, sensor based robot suggests that the costs of manual inspection can be reduced using automation. 
Whilst the exact location and shape of defects was not always clearly displayed, the sensor array did detect all the defects in the replica rail section. It also gives a good indication of depth. At a sensor height of approximately $0.6 \mathrm{~mm}$ from the rail surface the processed data is easy to interpret and indicates where further maintenance is needed.

The project has successfully built on the paper "Sensor Development and Application on the oil-gas pipeline Magnetic Flux Leakage Detection" [4], making a Hall effect sensors for NDT application. The optimising of the sensor systems explored in their paper have been successfully modified for use testing railway track. Additionally, the work has included the development of software to visualise the sensor data.

\section{References}

[1] M. Shaw, "Rail Trends Factsheet 2016," Gov.uk, 2017.

[2] Office of Rail and Road, "UK rail industry financial information 2016$17, " 2018$.

[3] D. F. Cannon, K.-O. Edel, S. L. Grassie, and K. Sawley, "Rail defects: an overview," Fatigue Fract. Eng. Mater. Struct., vol. 26, no. 10, pp. 865887, 2003.

[4] P. Lad and M. Pawar, "Evolution of Railway Track Crack Detection System," in 2nd IEEE International Symposium on Robotics and Manufacturing Automation, 2016.

[5] Lidén, T. and Joborn, M. (2016) 'Dimensioning windows for railway infrastructuremaintenance: Cost efficiency versus traffic impact', Journal of Rail Transport Planning \& Management, 6(1), pp. 32-47.

[6] Lijian, Y. et al. (2009) 'Sensor Development and Application on the oilgas pipeline Magnetic FluxLeakage Detection', The Ninth International Conference on Electronic Measurement \& Instruments. doi: 10.1109/ICEMI.2009.5274410.

[7] Mahfuz, N. et al. (2017) 'Autonomous Railway Crack Detector Robot for Bangladesh', in 2017 IEEE Region 10 Humanitarian Technology Conference. American International University - Bangladesh. doi: 10.1109/R10-HTC.2017.8289014.

[8] Misron, N. et al. (2011) 'A Mobile Ferromagnetic Shape Detection Sensor Using a Hall Sensor Array and Magnetic Imaging', Sensors, 11, pp. 10474-10489. doi: 10.3390/s111110474. 